\title{
Computer-aided design of ATO speed commands according to energy consumption criteria
}

\author{
M. Domínguez ${ }^{1}$, A. Fernández ${ }^{1}$, A. P. Cucala ${ }^{1}$ \& L. P. Cayuela ${ }^{2}$ \\ ${ }^{1}$ Instituto de Investigación Tecnológica, Escuela Técnica Superior de \\ Ingeniería (ICAI), Universidad Pontificia Comillas, Madrid, Spain \\ ${ }^{2}$ Dirección de Ingeniería, Metro de Madrid, Spain
}

\begin{abstract}
Traffic regulation systems of metro lines equipped with Automatic Train Operation (ATO) use a set of pre-programmed speed commands selecting coasting points and brake deceleration. Different speed commands provides different travel times between stations and the regulation system on-line selects and sends to the train one of these commands. Nowadays, speed commands are designed based on time and comfort criteria. In this paper a new approach of speed commands design, which takes into account not only present operational criteria but also energetic ones, is proposed in order to obtain energy efficient ATO commands. Firstly, the travel time and energy consumption of every command is calculated using a simulator that combines all the possible discrete values of the ATO configuration parameters. A set of systematic rules has been defined to include the consumption, operative and comfort criteria in the selection of the speed commands applying decision theory techniques. A software tool has been implemented for a computer-aided design of the speed commands. This tool includes a thorough simulation module of the train movement (ATO, motor and train dynamics), an automatic generator of every possible command and a graphical assistant for the speed commands selection according to the mentioned rules. The methodology described in this paper has been used to redesign the current ATO commands (4 for each station) of Line 1 of Madrid Underground. The results are presented in this paper. According to the simulation results, about $10 \%$ of energy savings are expected to be achieved with these new speed commands.
\end{abstract}

Keywords: energy consumption, speed commands design, train simulation. 


\section{Introduction}

Metro lines equipped with Automatic Train Operation systems (ATO) use preprogrammed speed commands to control the circulation of trains, providing a set of alternative ATO speed profiles per inter-station. As a result, driving is not influenced by human factors and run-times and energy consumptions are quite stable when signalling systems do not affect the circulation of trains. Traffic regulation systems performance and total energy consumption strongly depend on the off-line design of the ATO speed commands.

The ATO speed profile to be executed between two stations is on-line selected by the regulation system according to the required run-time. When a train must be held up, from the user's point of view a longer run-time is preferred rather than a longer station waiting time. In addition, this regulation strategy involves energy saving because longer run-times are obtained with slower speed profiles. However, these ATO speed profiles are usually designed according to run-time and comfort criteria, but not to energy consumption.

There are two main approaches to optimise train driving speed profiles: mathematical optimisation models and computer-aided design based on detailed simulation and direct search techniques.

The first approach includes in a mathematical model those restrictions that govern the train movement, such as track geometry, traction equipment, speed restrictions and driving rules. For instance Howlett [1] and Khmelnitsky [2] apply optimal control techniques for determining the optimal switching times in manual driving. The formulation and resolution of these analytical models require very complex techniques as well as important simplifications of the train dynamics or the driving strategy.

On the other hand, approaches based on simulation do not require simplifications and enable an accurate calculation of run-times and energy consumption, as Lukaszewicz manual driving modelling [3]. To explore the solution space and select alternative driving, different direct search methods are used, for instance heuristic search for ATO speed commands design [4] or genetic algorithms and fuzzy logic for manual and automatic driving optimisation [5], or genetics for optimisation of coasting points [6, 7]. Wong and Ho [8] compare different search methods for the on-line control of a train using an accurate simulator, determining the coasting points. This work stresses the importance of an accurate train movement modelling for practical applications.

The work presented in this paper is focussed on the computer-aided design of the ATO speed commands between two metro stations, to be pre-programmed in the ATO equipment. The variables to optimise will be the configuration data of each particular ATO system, four parameters in the case study of Line 1 of Madrid Underground: coasting, re-motoring and regulation velocities, and braking deceleration rate. The considered ATO system provides only certain discrete values for each parameter, resulting a solution space of 220 alternative speed profiles per inter-station in Madrid Underground, and this fact allows the exhaustive and accurate simulation of the whole feasible ATO speed profiles. Instead of search techniques, decision theory techniques can be directly applied 
to select a set of solutions per inter-stations (4 in the case study) including operational and energy consumption criteria. This way, the obtained driving solutions will be fully adjusted to real features and capabilities of the ATO equipment in service.

A software tool for computer-aided design of ATO speed profiles has been developed, to support the design procedure defined. Next sections will describe briefly the simulator, the proposed design procedure and the simulation results of its application to Line 1 of Madrid Underground. The obtained solutions are compared with the current driving profiles in service in this line in order to value the expected energy savings.

\section{The simulator}

The proposed design method is based on the accurate simulation of energy consumption and run-time of all the possible combinations of ATO speed commands for each inter-station. Train velocity, acceleration, traction or brake force and energy consumption are computed at each simulation step. The speed/distance profile between stations is plotted to assist the design process.

The simulator is composed of three modules: ATO equipment simulator, train dynamics model and train consumption model. This modular architecture allows the validation of each module separately and an easy adjustment for specific features of a particular ATO equipment. To this end, the simulator input interfaces are designed to enable the definition of track layout, train characteristics, and ATO system configuration.

\subsection{ATO equipment simulator}

This module calculates the type of motion at each simulation step: motoring, coasting or braking. The particular ATO system modelled in the case study (Line 1 of Madrid Underground) supplies a unique traction command (the maximum). In contrast, there are four values for deceleration braking command which can be selected. The regulation speed order is implemented by means of maximum traction and coasting cycles in ramps (or braking and coasting cycles in slopes). This control is also applied when the train reaches the maximum speed of the track.

The ATO simulator is configured with the real fixed and variable parameters needed for an accurate simulation. The fixed parameters are:

- Safety distance: to be observed when the train has to brake due to a maximum speed reduction.

- ATP safety offset: to be observed under the maximum speed

- Positive and negative regulation offsets: The regulation speed cycles previously mentioned are applied between the speed limits defined by these offsets.

On the other hand, the variable parameters (speed commands to be designed) are:

- $\quad$ Braking command: deceleration rate during the braking process. 
- $\quad$ Regulation speed. The target speed of the train is the minimum between the maximum velocity and the regulation one.

- Coasting speed: When reached, traction is turned off up to re-motoring speed.

- Re-motoring speed: When reached, traction is turned on up to coasting speed.

The ATO simulator model also includes the necessary rules and algorithms to emulate the real operation of the equipment calculating:

- Coasting and re-motoring points: where the traction is turned off or turned on.

- $\quad$ Starting braking points. The actual ATO braking algorithms are replicated to decide at each simulation step if the train has to brake to observe a speed reduction or a stopping point.

\subsection{Train dynamic model}

$$
a=\frac{\sum\left(F-F_{g}-F_{a}\right)}{M_{I}}
$$

This module recalculates train speed and position at each simulation step. They are obtained from the train acceleration $a$, which is the result of the traction force $F$ minus the rolling and aerodynamic resistance to train movement $F_{a}$ and track gradient and curvature resistances $F_{g}$ as shown in eq

(1). $M_{I}$ stands for train mass plus the rotational inertial effect.

The traction motor curve (maximum force/speed) is given as input data in order to calculate $F$. Power and grip limitations are taken into account in this curve. The resistance to train movement $F_{a}$ is modelled as a quadratic function of the velocity with nonnegative parameters depending on each particular train. The track gradient resistance $F_{g}$ is the resistive force due to gravity, positive for ramps and negative for slopes.

While the train is braking, the ATO system regulates the force continuously in order to obtain the braking command reference. The simulator applies precisely this deceleration rate, assuming that the train is able to supply the needed braking force.

\subsection{Consumption model}

The energy consumption $E$ is recalculated according to the time increment $\Delta t$ and the current $I$ at each simulation step. The current is obtained from the line current/speed curve. A constant line voltage $U$ is assumed.

$$
E=I \cdot U \cdot \Delta t
$$

\subsection{Operational and comfort restrictions}

After simulating every ATO speed profile, a validation model checks the fulfilment of the operational and comfort restrictions specified. In the study case 
of Madrid Underground, the current restrictions are the minimum speed along curves (avoiding to wear the track) and the jerk restriction for a comfortable trip.

\section{ATO speed commands design and case study}

Once inter-station run-times and energy consumption have been simulated for all the possible ATO speed profiles, a set of them are selected to be programmed in the ATO equipment.

Decision theory techniques have been used to solve this multicriteria problem finding an appropriate trade-off between costs (energy consumption) and runtimes because the longer the run-time is, the lower the energy consumption is. According to that, the optimal solutions are over the Pareto curve which represents the minimum consumption for each run-time. An example is given in Figure 1.

The proposed procedure follows three criteria: domination, sensitivity and uniform distribution of run-times. They have been applied to optimize the speed commands design of the Line 1 of Madrid Underground. The following description of the procedure will be illustrated with this realistic application. The energy consumption and run-times of the current speed profiles and the proposed ones will be compared in order to value the achievable energy saving.

In Madrid Underground there are four alternative speed profiles per interstation to be designed, with increasing run-times from the first (flat out, the fastest) to the fourth (slowest). The first speed profile is obtained applying maximum speed and deceleration conditions.

\subsection{Domination criterion}

According to this criterion, optimal solutions are over the Pareto curve which is formed by the solutions with less energy consumption and approximately the same run-time of all the possible ones. Solutions not located on the curve are said to be dominated and are discarded.

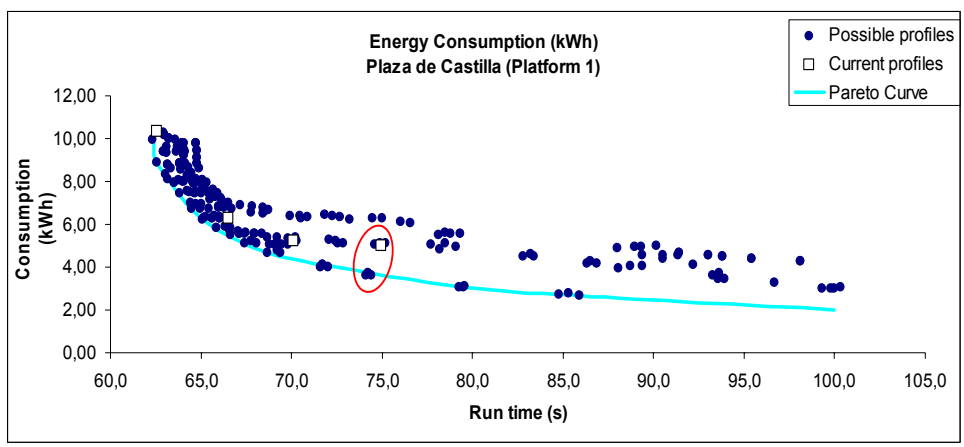

Figure 1: Current speed profiles dominated by others with less consumption and Pareto curve. 
An example of the case study is given in Figure 1 (from Plaza de Castilla station to Valdeacederas station). The marked $4^{\text {th }}$ speed profile currently in service, is dominated by two others over the Pareto curve with almost the same run-time. The proposed solution consumes $28 \%$ less energy than the current one (Table 1).

Table 1: $\quad 28 \%$ energy savings with an alternative speed profile.

\begin{tabular}{|c|c|c|c|c|c|}
\cline { 2 - 6 } \multicolumn{1}{c|}{} & Braking & Coasting & Motoring & Time & Consumption \\
\hline Profile & $\mathrm{m} / \mathrm{s} 2$ & $\mathrm{~km} / \mathrm{h}$ & $\mathrm{km} / \mathrm{h}$ & $\mathrm{s}$ & $\mathrm{kWh}$ \\
\hline Current & 0.65 & 32 & 10 & 75.0 & 4.99 \\
\hline Alternative & 0.7 & 35 & 20 & 74.4 & 3.6 \\
\hline
\end{tabular}

\subsection{Consumption sensitivity criterion}

This is the criterion to be applied for the slowest speed profile selection.

In the time/consumption graph, the slope of the Pareto curve progressively decreases from the fastest speed profile as the run-time increases. That is to say, solutions near the minimum run-time speed profile have high marginal energetic cost associated per second. These marginal costs go down reaching almost 0 at the end of the curve. The proposed criterion places the slowest speed profile where the Pareto slope becomes almost flat. This strategy guarantees energy savings when trains are held up for traffic regulation purposes.

The maximum run-time gap between the fastest and the slowest speed profile is limited in practice by operational requirements, so the slowest profile must be moved and placed before the flat slope of the curve if it is necessary to observe this restriction. Figure 2 shows an example of the case study (from Tetuán to Estrecho stations). The Pareto curve becomes flat for run-times greater than 140 $\mathrm{s}$, and the fastest one takes $70 \mathrm{~s}$. However, the maximum run-time gap defined for Madrid Underground is $20 \mathrm{~s}$. Therefore, the fourth speed profile must take less than $140 \mathrm{~s}$ and it is placed $20 \mathrm{~s}$ slower than the fastest one, observing the operational requirements. This selection involves that the fourth profile can be 7 s slower than the current one, and this implies $22 \%$ of energy savings (Table 2 ).

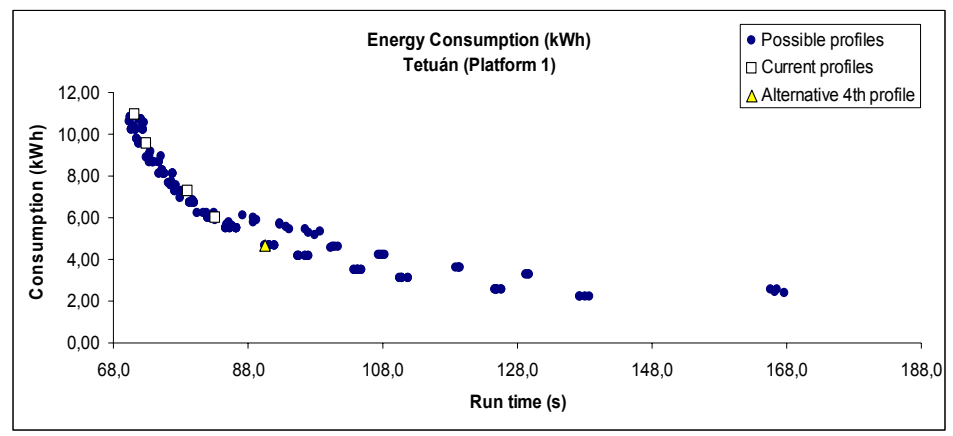

Figure 2: $\quad$ Alternative $4^{\text {th }}$ speed profile with less consumption. 
Table 2: $\quad$ An alternative speed profile 7 s slower achieves $21.7 \%$ of savings.

\begin{tabular}{|c|c|c|c|c|c|}
\cline { 2 - 6 } \multicolumn{1}{c|}{} & Braking & Coasting & Motoring & Time & Consumption \\
\hline Profile & $\mathrm{m} / \mathrm{s} 2$ & $\mathrm{~km} / \mathrm{h}$ & $\mathrm{km} / \mathrm{h}$ & $\mathrm{s}$ & $\mathrm{kWh}$ \\
\hline Current & 0.65 & 42 & 10 & 83.1 & 5,98 \\
\hline Alternative & 0.75 & 37 & 30 & 90.5 & 4.68 \\
\hline
\end{tabular}

\subsection{Temporal uniform distribution criterion}

Once the fastest and the slowest speed profiles have been selected in accordance with the previous criteria, the remaining profiles are designed applying the uniform distribution criterion. The speed commands must be selected in order to obtain a design with a uniform distribution of the run-times over the Pareto curve. This design allows a proper operation of the traffic regulation system.

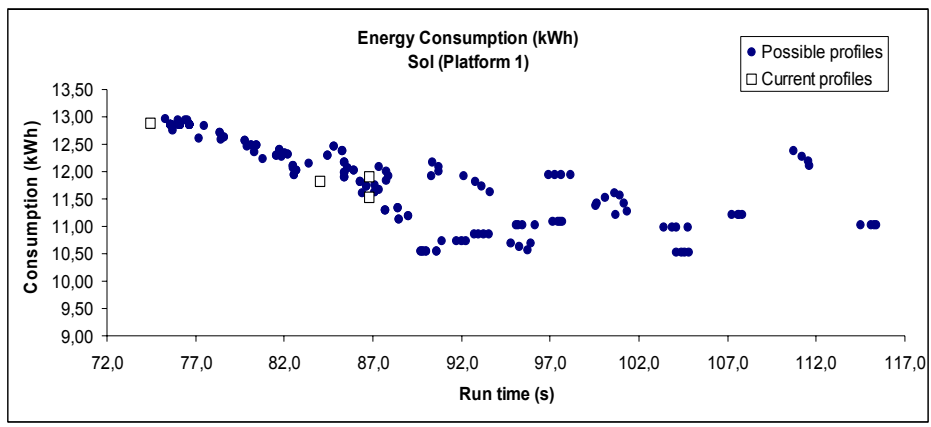

Figure 3: Current speed profiles.

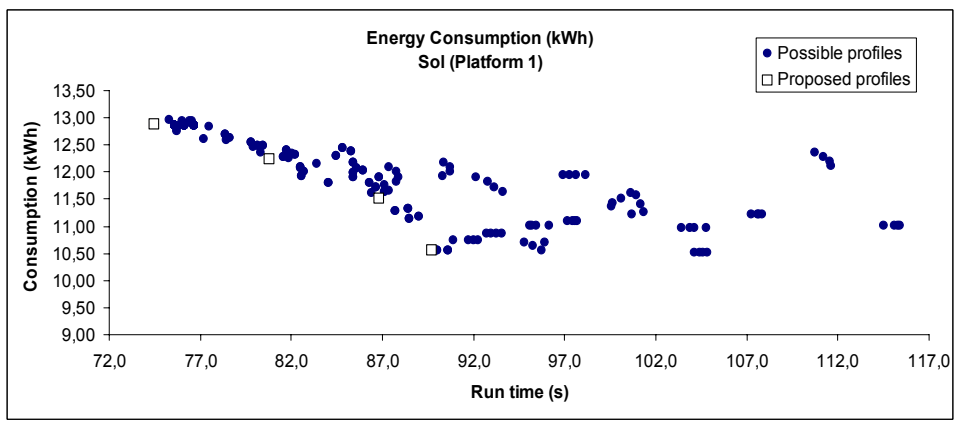

Figure 4: $\quad$ Proposed speed profiles designed.

In the study case, the uniform distribution criterion is applied to design two profiles (the second and the third) between the two profiles previously selected. Figure 3 shows the current profiles in service between Sol and Tirso de Molina stations. In the proposed design shown in Figure 4, second and third profiles are selected to obtain time differences of $5 \mathrm{~s}$ approximately. 


\subsection{Redesign of the current ATO speed commands of the Line 1 of Madrid Underground}

The design procedure has been applied to redesign the ATO speed commands of all the trips between the 26 stations of Line1 of Madrid Underground in order to value the expected energy savings. The discrete possible values of the ATO equipment parameters in service are shown in Table 3. The combination of them supplies 220 possible speed profiles to be simulated between each two stations. The average simulation time for these 220 profiles, for one inter-station, is $4 \mathrm{~s}$.

Table 3: Configuration parameters of the ATO in service.

\begin{tabular}{|c|c|c|c|c|c|c|c|c|c|c|c|c|c|c|c|c|c|}
\hline Braking command & $(\mathrm{m} / \mathrm{s} 2)$ & 0,8 & 0,75 & 0,7 & 0,65 & & & & & & & & & & & & \\
\hline Regulation Speed & $(\mathrm{km} / \mathrm{h})$ & 62 & 60 & 57 & 55 & 52 & 50 & 47 & 45 & 42 & 40 & 37 & 35 & 32 & 30 & 27 & 25 \\
\hline Coasting speed & $(\mathrm{km} / \mathrm{h})$ & 57 & 55 & 52 & 50 & 47 & 45 & 42 & 40 & 37 & 35 & 32 & 30 & 27 & 25 & 22 & 20 \\
\hline Remotor speed & $(\mathrm{km} / \mathrm{h})$ & 30 & 20 & 10 & & & & & & & & & & & & & \\
\hline
\end{tabular}

Table 4: $\quad$ Summary table. Average energy savings and run-time increase in the proposed speed profiles in Line 1.

\begin{tabular}{|c|c|c|c|c|c|}
\cline { 2 - 6 } \multicolumn{1}{c|}{} & Designed Profiles & \multicolumn{2}{c|}{ Current Profiles } & \multicolumn{2}{c|}{ Diferences } \\
\cline { 2 - 6 } \multicolumn{1}{c|}{} & Consumption & Time & Consumption & Time & Consumption \\
\hline Profile & $\mathrm{kWh}$ & $\mathrm{s}$ & $\mathrm{Km} / \mathrm{h}$ & $\%$ & $\%$ \\
\hline 1 & 225.4 & 1665.5 & 252.4 & 0.5 & 10.7 \\
\hline 2 & 176.3 & 1794.6 & 196.5 & -0.4 & 10.3 \\
\hline 3 & 147.0 & 1915.8 & 159.1 & 0.6 & 7.6 \\
\hline 4 & 128.3 & 2032.5 & 145.9 & 1.4 & 12.1 \\
\hline & 677.0 & 7408.5 & 753.9 & 0.5 & 10.2 \\
\hline
\end{tabular}

The simulation results show that $70 \%$ of the redesigned speed profiles save more than $10 \%$ of energy, while $26 \%$ of them save more than $20 \%$. Overall, an average of $10 \%$ energy savings is expected increasing the run-times only $0.5 \%$ as Table 4 shows. Moreover, not only an energy benefit is obtained, but also speed profiles are time-uniformly distributed.

\section{New ATO configuration parameters}

Although the new speed profiles for Line 1 have been designed according to the current possibilities of the ATO equipment, the simulator allows researching and justifying further modifications and improvements for future ATO systems. This is another advantage of a computer-aided design based on accurate simulation.

In Madrid Underground the ATO system applies a unique set of speed commands for each speed profile between two stations that is executed during the whole trip. A set is composed of a brake deceleration and a pair of coasting and motoring or a regulation speed command. However, being possible to define different sets for a single trip so that they could be on active in particular sections of the trip, more alternative speed profiles would be obtained. In particular, defining the activation of a coasting command set at the end of the trip, just before the final breaking, it is possible to replace braking by coasting. For 
instance, the feasible profiles considering the ATO in service in Line 1 between Sol and Tirso de Molina stations are plotted in Figure 3. In Figure 5, new speed profiles are obtained defining different activation sections for the coasting commands sets. The number of solutions below the previous Pareto curve rises notably. In particular, the third profile could be redesigned, saving $17 \%$ of energy with the same run-time. These speed profiles are shown in Figure 6.

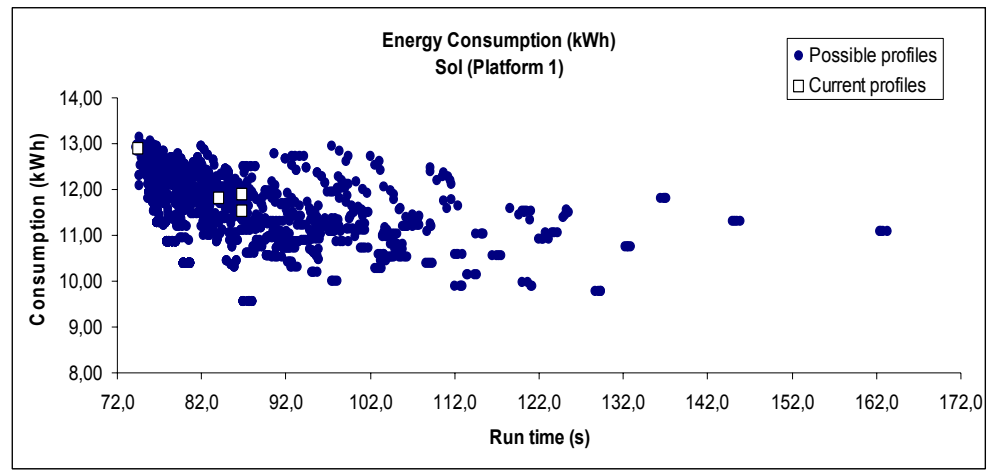

Figure 5: $\quad$ Simulated speed profiles with a starting point of commands set.

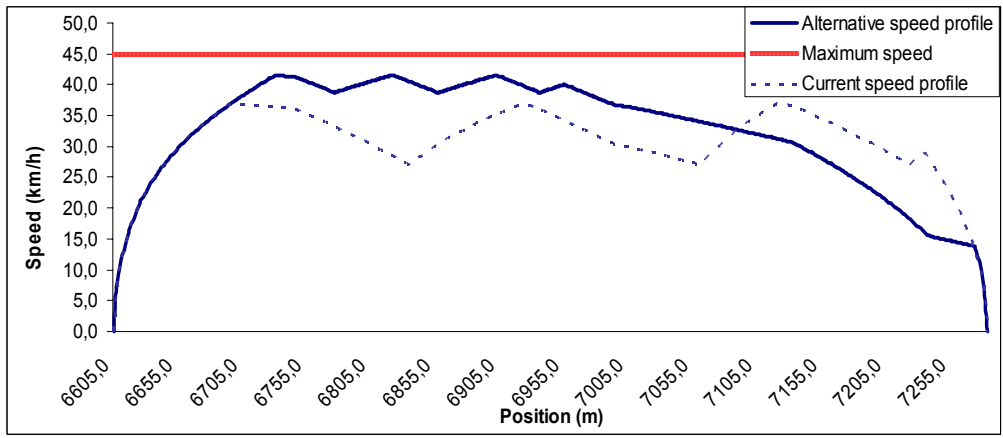

Figure 6: Comparison between the current speed profile and an alternative one with a starting point of commands set.

\section{Conclusions}

The study shows the importance of a detailed model of the particular ATO system in order to obtain a realistic simulation that allows calculating slight differences between alternative speed profiles. In the case study of Madrid Underground, these differences can be a few seconds.

In addition, current ATO equipments provide only a certain range of discrete configuration values. Thus, there is a finite and relatively short number of possible speed profiles that can be exhaustively simulated. Decision theory 
techniques can be directly applied to select solutions finding a trade-off between run-times and energy costs.

From the redesign of the ATO speed commands of Line 1 of Madrid Underground it is possible to conclude that an improvement of about $10 \%$ of energy consumption is expected without degrading run-times. Even $20 \%$ of savings are expected with $26 \%$ of the redesigned speed profiles. In high frequency metro lines the programmed speed profiles are repeated systematically many times, so the energy savings become a relevant aspect.

Finally, the proposed design procedure based on simulation allows the study of new features and configuration parameters to be implemented in future ATO equipments, with the aim of obtaining more energy efficient speed profiles, as it has been shown in the case study.

\section{References}

[1] P. Howlett, "The Optimal Control of a Train," Annals of Operations Research, vol. 98, pp. 65, 2000.

[2] E. Khmelnitsky, "On an Optimal Control Problem of Train Operation," IEEE Transactions on Automatic Control, vol. 45, pp. 1257, 2000.

[3] P. Lukaszewicz, "Energy Consumption and Running Time for Trains," in KTH, Department of Vehicle Engineering. Royal Institute of Technology, Stockholm, 2001, pp. 153.

[4] F. de Cuadra, A. Fernandez, J. de Juan, and M. A. Herrero, "Energy-saving automatic optimisation of train speed commands using direct search techniques," in Computers in Railways V - Vol.1 Railway Systems and Management, 1996, pp. 337-346.

[5] Y. V. Bocharnikov, A. M. Tobias, C. Roberts, S. Hillmansen, and C. J. Goodman, "Optimal driving strategy for traction energy saving on DC suburban railways," IET Electric Power Applications, vol. 1, pp. 675, 2007.

[6] H. Seong Ho, B. Yun Sub, B. Jong Hyen, A. Tae Ki, L. Su Gil, and P. Hyun Jun, "An optimal automatic train operation (ATO) control using genetic algorithms (GA)," in Proceedings of IEEE. IEEE Region 10 Conference. TENCON 99. 'Multimedia Technology for Asia-Pacific Information Infrastructure' (Cat. No.99CH37030), vol.1.

[7] C. S. Chang and S. S. Sim, "Optimising train movements through coast control using genetic algorithms," IEE Proceedings-Electric Power Applications, vol. 144, pp. 65, 1997.

[8] K. K. Wong and T. K. Ho, "Coast control for mass rapid transit railways with searching methods," IEE Proceedings-Electric Power Applications, vol. 151, pp. 365, 2004. 